\title{
Análise da velocidade e acurácia de movimentos em indivíduos após acidente vascular encefálico
}

\section{Speed and accuracy of movement in individuals after stroke}

Gabriela Araújo $^{1}$, Nataly Rossini ${ }^{1}$, Aline Sanchez Ferrari ${ }^{1}$, (DCarlos Bandeira de Mello Monteiro ${ }^{2}$, (DTalita Dias da Silva ${ }^{2}$, (DThais Massetti ${ }^{2}$, Deborah Cristina Gonçalves Luiz Fernani ${ }^{1}$, (D) Maria Tereza Artero Prado Dantas ${ }^{1}$

\footnotetext{
1 Universidade do Oeste Paulista - UNOESTE

2 Escola de Artes, Ciências e Humanidades da

Universidade de São Paulo, Grupo de Pesquisa e Aplicação Tecnológica em Reabilitação - PATER

Correspondência

Maria Tereza Artero Prado Dantas

E-mail: mariatereza@unoeste.br
}

Submetido: 30 Novembro 2018

Aceito: 17 Junho 2020

Como citar

Araújo G, Rossini N, Ferrari AS, Monteiro CBM, Silva TD, Massetti T, et al. Análise da velocidade e acurácia de movimentos em indivíduos após acidente vascular encefálico. Acta Fisiatr. 2020;27(2):100-106

DOI: 10.11606/issn.2317-0190.v27i2a172968

\section{cc) $\$$}

\section{RESUMO}

O Acidente Vascular Encefálico (AVE) pode causar alteração no controle dos movimentos, devido as sequelas deste processo. Objetivo: Analisar a relação entre a velocidade e acurácia de movimento de membro superior em indivíduos com sequela de AVE com um software computacional. Método: Trata-se de um estudo transversal, a amostra foi composta por 46 indivíduos divididos em dois grupos: 24 indivíduos com AVE, com idade entre 35 e 83 anos, sendo 10 mulheres e 14 homens; e 22 Controles saudáveis pareados por idade e sexo, sendo 8 mulheres e 14 homens, avaliados por meio dos instrumentos: Mini Exame do Estado Mental, Escala Prognóstica de Orpington, Escala de Fulg Meyer, dinamômetro, caixa de blocos e pelo software "Fitts Reciprocal Aiming Task v.2.0 (Horizontal)", que busca compreender o controle motor do membro superior, verificando a velocidade e acurácia do movimento, por uma tarefa de computador. Resultados: Os indivíduos com sequela de AVE apresentaram prejuízo da força de preensão palmar e da função manual, além de maior tempo de movimento em todos os índices de dificuldade comparados ao grupo controle. No entanto, estes apresentaram comportamento semelhante aos indivíduos saudáveis ao longo da realização dos índices de dificuldade. Conclusão: Portanto, pode-se concluir que os indivíduos com sequela de AVE apresentaram déficit do controle motor de membro superior, comparado a indivíduos saudáveis, porém estes apresentaram o mesmo comportamento, com déficit maior na acurácia do movimento.

Palavras-chave: Acidente Vascular Cerebral. Destreza Motora. Análise e Desempenho de Tarefas

\section{ABSTRACT}

The Stroke might cause alterations in movement control due to the sequels of this process. Objective: Analyze the speed-accuracy trade-off of upper limb movement in individuals with sequels of Stroke through a computer software. Method: It is about a cross-sectional study, the sample was composed of 46 individuals divided into two groups: individuals post-stroke, between the ages of 35 and 83 years old, in which 10 were women and 14 were men; and 22 healthy controls aligned by age and sex, in which 8 were women and 14 were men, evaluated through the instruments: Mini mental state examination, Orpington prognostic scale, Fugl-Meyer Assessment Scale, dynamometer, box of blocks and throught the software "Fitts Reciprocal Aiming Task v.2.0. (Horizontal)", that seeks to understand the motor control of the upper limb, verifying the speed and accuracy of movement through a computer task. Results: The individuals with sequels of Stroke showed a loss in the strength of the palmar grip and manual function. Besides, they showed a longer time in movement in all index of difficulty compared to Control group. However, they showed a similar behaviour to the healthy individuals throughout the execution of the index of difficulty. Conclusion: Thus, it can be concluded that the individuals with sequels of Stroke showed a deficit of the motor control of the upper limb, compared to the healthy individuals, however, these showed the same behavior, with a bigger deficit in the accuracy of movement.

Keywords: Stroke. Motor skills. Task Performance and Analysis 


\section{INTRODUÇÃO}

O Acidente Vascular Encefálico (AVE) trata-se da falta de suprimento sanguíneo adequado ao encéfalo, sendo este de causa isquêmica ou hemorrágica. ${ }^{1,2}$ No Brasil e em diversos países desenvolvidos, o AVE é responsável pelos maiores casos de incapacidades. De acordo com a Organização Mundial da Saúde, a cada 100 mil habitantes, 108 destes poderão ter algum tipo de sequela. ${ }^{3-7}$ Nos EUA a cada 40 segundos uma pessoa pode ter um AVE, e por ano o número total pode chegar a 750 mil pessoas. ${ }^{8,9}$

A sequela mais comum do AVE, é a hemiparesia, que desencadeia diversos acometimentos, sendo um destes a redução da independência no controle isolado dos dedos, que podem diminuir a velocidade e destreza das mãos e consequentemente a habilidade motora fina, ${ }^{10-15}$ e como esta, é fundamental nas atividades de vida diária, as consequências do AVE trazem prejuízo na execução de tarefas simples. ${ }^{16}$

Os distúrbios da função motora após o AVE são atribuídos aos seguintes déficits: redução da movimentação corticoespinal aos músculos agonistas, atividade reflexa alterada, espasticidade e comprometimento da coordenação motora. ${ }^{17}$ Quanto maior a velocidade dos estímulos conduzidos ao cérebro, mais informações serão absorvidas rapidamente e consequentemente, a resposta frente à essa informação será com maior acurácia. Um estudo ${ }^{18}$ indicou que a precisão, é um dos fatores a serem considerados na melhoria do desempenho motor durante a manipulação das mãos de pacientes.

Alguns trabalhos demonstraram o crescimento do uso de tarefas de computador na avaliação motora fina em indivíduos com sequela neurológica, ${ }^{19-22}$ para verificação da aprendizagem motora ou do controle motor, a partir da relação entre a velocidade e acurácia do movimento. Porém, ainda é pequeno o relato de estudos com este tipo de avaliação em AVE, sendo necessária a abordagem desta avalição.

Sendo a hipótese deste estudo, que indivíduos com sequela por AVE apresentariam maior dificuldade para a realização de tarefas que exigiam velocidade e principalmente acurácia do movimento, comparando-se com indivíduos sem sequela com mesma idade e sexo.

\section{OBJETIVO}

O objetivo deste estudo foi analisar a relação entre a velocidade e acurácia de movimento de membro superior em indivíduos com sequela de AVE com um software computacional.

\section{MÉTODOS}

Trata-se de um estudo transversal aplicado em uma clínica de fisioterapia de Presidente Prudente/SP, aprovado por comitê de ética e pesquisa (com número de CAAE: 63123116.7.0000.5515), que analisou a velocidade e acurácia do movimento de membros superiores de indivíduos com sequela de AVE, utilizando uma tarefa de computador baseada na Lei de Fitts, comparando-os com indivíduos sem sequela.

\section{Participantes}

Foram avaliados 46 indivíduos, divididos em dois grupos:
- Grupo AVE com 24 indivíduos com sequela de AVE, com idade entre 35 e 83 anos (com média de idade de $57 \pm 12$ anos), sendo 10 mulheres e 14 homens;

- Grupo Controle com 22 sujeitos saudáveis (sem sequelas neurológicas) pareados por idade e sexo ao Grupo AVE (dois sujeitos foram excluídos deste grupo durante as análises dos dados, por se tratarem de "outliers"), sendo 8 mulheres e 14 homens.

Como critérios de inclusão, os indivíduos do Grupo AVE deveriam ter sequela de AVE de no mínimo 2 meses pós lesão e com adequada função mental e cognitiva, verificada pelo Mini Exame de Estado Mental (MEEM). ${ }^{23}$ Já os critérios de exclusão do estudo foram a presença de alterações visuais, deformidades e desbloqueio neuromuscular recente (últimos seis meses).

\section{Materiais e procedimentos}

Em relação a caracterização do Grupo AVE, os indivíduos foram avaliados pelo MEEM, como já relatado, e pela Escala Prognóstica de Orpington, ${ }^{24}$ que avalia o déficit motor, a propriocepção, equilíbrio e cognição, sendo que as pontuações neste instrumento variam de 0 (menor nível de incapacidade) para 5 (maior nível de deficiência).

Além destes serem avaliados pela Escala de Fulg-Meyer, ${ }^{25,26}$ que avalia a amplitude de movimento, sensibilidade, dor, equilíbrio, função motora de membro superior e inferior do indivíduo que sofreu AVE, sendo que quanto mais próximo de $100 \%$, melhor é a função sensório-motora destes. ${ }^{25,26}$

Além dos instrumentos citados, os indivíduos da amostra foram também avaliados com diversos outros, sendo estes: Caixa de Blocos para análise da função motora de membro superior; ${ }^{27}$ Dinamometria para verificação da força de preensão manual; ${ }^{28-30}$ "Fitts 'Reciprocal Aiming Task v.2.0 (Horizontal)"31 para avaliar o controle motor, pela relação entre velocidade e acurácia do movimento, que serão detalhados a seguir.

\section{Caixa de blocos}

O teste de caixa de blocos verificou a função motora de membro superior, a partir da facilidade e velocidade manual, ${ }^{27}$ sendo que esta avaliação foi descrita por Ayres et al.

A caixa de blocos apresentava as seguintes dimensões externas: $53,7 \mathrm{~cm}$ de comprimento, $25,4 \mathrm{~cm}$ de largura e bordas com 7,5 cm de altura, com uma divisória central vertical de $15,2 \mathrm{~cm}$ de altura que separa a caixa em dois compartimentos de tamanhos iguais, além da espessura da madeira de $1,0 \mathrm{~cm}$.

$\mathrm{Na}$ caixa haviam cento e cinquenta blocos de madeira em forma de cubos com $2,5 \mathrm{~cm}$ de lado nas cores primárias (vermelho, azul e amarelo), sendo 50 unidades de cada cor. ${ }^{32}$

Para realização deste teste os indivíduos (grupo controle e grupo AVE) permaneciam sentados frente a caixa de blocos, posicionada sobre a mesa, sendo solicitado que deslocassem o mais rápido possível cada bloco (independentemente de cor) para o lado oposto ao membro em uso para a tarefa.

O tempo para realização da tarefa era de um minuto, uma única vez para cada membro, sendo registrado a quantidade de blocos deslocados neste intervalo de tempo. 


\section{Dinamometria}

Foi utilizado neste estudo o dinamômetro de sistema hidráulico $\left(\right.$ Jamar $\left.^{\circledR}\right)$ para realizar a mensuração da força de preensão manual. ${ }^{28-30}$ Neste teste o ombro do indivíduo era mantido em posição neutra, com cotovelo fletido a $90^{\circ}$, punho estendido entre 0 e $30^{\circ}$ e desvio ulnar de 0 a $15^{\circ},{ }^{33}$ sendo solicitado ao indivíduo que pressionasse o instrumento com a maior força possível. Foram realizados três testes, com intervalo de um minuto entre eles, sendo realizado a média entre os três valores obtidos para posterior análise.

\section{Fitts Reciprocal Aiming Task v.2.0 (Horizontal)}

O software Fitts Reciprocal Aiming Task v.2.0 (Horizontal) desenvolvido por Okazaki ${ }^{31}$ e utilizado neste estudo, segue o método de avaliação pela Lei de Fitts, $^{34}$ o qual busca compreender o sistema de controle motor do membro superior, a partir da relação entre a velocidade e a acurácia do movimento, que é determinada pela relação log-linear entre o tamanho do alvo e distância entre estes, ${ }^{19}$ resultando no índice de dificuldade (ID). ${ }^{34}$

Para aplicação do software foi utilizado um computador da marca HP Pavillion ${ }^{\circledR} \mathrm{dm} 4$, modelo $\mathrm{dm} 4-2075 \mathrm{br}$ e um mouse optical 5.0 da marca Fortrek $^{\circledR}$, sendo que para realização da tarefa o indivíduo foi posicionado em uma cadeira frente a mesa com altura adequada.

A tarefa era composta por duas barras verticais (alvos) na tela do computador, que os indivíduos deveriam clicar com o mouse alternadamente e o mais rápido possível, após indicação sonora de início. As barras mudavam de largura e distância conforme o ID, sendo necessário realizar três tentativas seguidas com um mesmo ID, anotando o tempo total de toques feitos pelo indivíduo avaliado por um tempo determinado de 10 segundos.

Foi utilizado neste estudo quatro índices de dificuldade, sendo estes o ID2, ID4a, ID4b e ID6. O ID2 era composto por barras verticais pareadas mais espessas $(3 \mathrm{~cm})$, com pouca distância entre estas $(6 \mathrm{~cm})-(\log 2[(2 \times 6) / 3]=\log 24=2)$, o ID4a formado por barras verticais laterais mais espessas $(3 \mathrm{~cm})$ com distância maior entre estas $(24 \mathrm{~cm})-(\log 2[(2 \times 24) / 3]=$ Log2 $16=4)$, o ID4b dispõe de barras laterais mais finas do que utilizado no ID4a $(1,5 \mathrm{~cm})$, porém, com a distância reduzida entre eles $(12 \mathrm{~cm})-(\log 2[(2 \times 12) / 1,5]=\log 216=4)[30-31] \mathrm{e}$ o ID6 formado por barras mais finas $(1,5 \mathrm{~cm})$ e mais distantes $(48 \mathrm{~cm})-(\log 2[(2 \times 48) / 1,5]=\log 64=6)$.

Foram cumpridos todos os princípios éticos (proteger a vida, a saúde, a privacidade e a dignidade do ser humano) que estão contidos na Declaração de Helsinki (2000), além do atendimento a legislações específicas do Brasil onde a pesquisa foi realizada.

\section{Análise dos dados}

Em relação às comparações do tempo de movimento em cada ID, uma ANOVA com fator 2 (grupos: AVE e Controle) por 2 (ID) foi realizada, com repetidas medidas para o índice de fator de dificuldade. Para o fator ID, foram realizadas análises separadas considerando: ID2 versus ID4a, ID4a versus ID4b e ID4b versus ID6. O teste post hoc foi realizado usando TukeyHSD.
Uma análise de regressão linear múltipla considerando aumento no tempo de movimento ( $\Delta$ entre ID6 e ID2) foi realizada para determinar quais fatores (idade, sexo, lado da hemiparesia, tipo de lesão, tempo de lesão, Fugl-Meyer, MEEM, Orpington, Caixa de blocos - lados direito e esquerdo e Dinamometria - lados direito e esquerdo) influenciaram no aumento do tempo de movimento. O nível de significância adotado foi de $p<0,05$ e o programa estatístico utilizado foi o SPSS, 20.0.

\section{RESULTADOS}

Considerando as características dos sujeitos do Grupo AVE, 8 (33\%) apresentavam hemiparesia à direita e 16 (67\%) à esquerda, 5 (21\%) apresentaram AVE do tipo Hemorrágico e 19 (79\%) do tipo Isquêmico. O tempo de lesão destes variou entre 2 e 282 meses ( $423 \pm 65$ meses), com pontuações do MEEM que variaram entre 0 e 30 pontos ( $21 \pm 8$ pontos). Em relação a gravidade da lesão, mensurada pela escala de Orpington, variou entre 0 e 5 ( $3 \pm 1$ pontos), a funcionalidade avaliada pela escala de Fugl-Meyer que variou de 42 a 100 (73 \pm 15 pontos). Já os achados das avaliações feitas com a Caixa de blocos e a Dinamometria, estão representadas na Tabela 1.

\section{ID2 - ID4a}

Houve diferença estatisticamente significativa entre os índices de dificuldade $\left(\mathrm{F}_{1,43}=8,40 ; \mathrm{p}=0,006 ; \mathrm{\eta}^{2}=0,16\right)$. Este resultado demonstra que o tempo de movimento aumentou significativamente de ID2 (média= 2,520s) para ID4a (média= 3,281 s). No entanto, nenhuma interação foi encontrada entre os grupos e os índices de dificuldade. Além disso, foi encontrado um efeito para grupos $\left(\mathrm{F}_{1,43}=10,2 ; \mathrm{p}=0,003 ; \mathrm{\eta}_{p}{ }^{2}=\right.$ 0,19 ), no qual os indivíduos com AVE (média $=3,797 \mathrm{~s}$ ) foram $1,794 \mathrm{~s}$ mais lentos do que os indivíduos do grupo Controle média=2,003s). Os resultados estão representados na Figura 1.

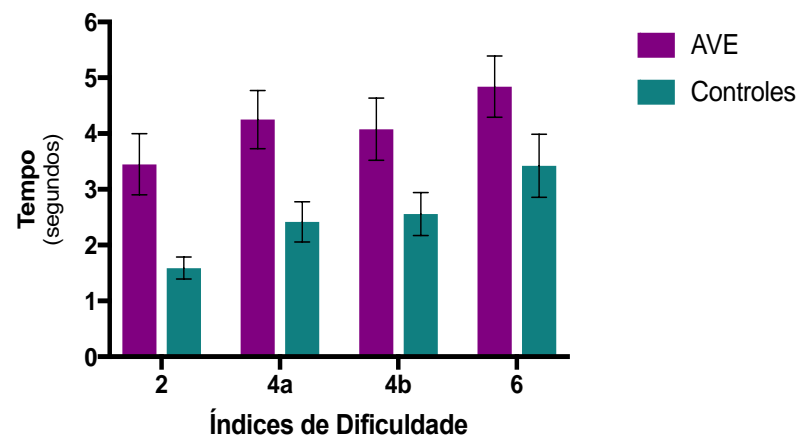

Figura 1. Valores de média e erro padrão em cada índice de dificuldade (ID) para ambos grupos

\section{ID4b - ID6}

De forma similar à comparação entre ID2 e ID4a, foi encontrada diferença estatisticamente significativa entre os índices de dificuldade $\left(F_{1,434}=9,29 ; \mathrm{p}=0,004 ; \eta_{p}{ }^{2}=0,17\right)$. Ou seja, houve aumento significativo de ID4b (média= 3,318s) para ID6 (média $=4,132 \mathrm{~s}$ ). No entanto, nenhuma interação foi encontrada entre os grupos e os índices de dificuldade. $O$ efeito para grupos se manteve presente $\left(\mathrm{F}_{1,44}=4,52 ; \mathrm{p}=0,039 ; \mathrm{\eta}^{2}=\right.$ $0,09)$. Este resultado mostra que os indivíduos com AVE 
(média $=4,460$ s) foram 1,470 s mais lentos do que os indivíduos do grupo Controle (média= 2,990s). Os resultados estão representados na Figura 1.
A análise de regressão não encontrou nenhum efeito significante para a influência dos fatores avaliados sobre o aumento do tempo de movimento nos índices de dificuldade.

Tabela 1. Média dos testes caixa de blocos e dinamometria para os grupos AVE e Controle

\begin{tabular}{|c|c|c|c|}
\hline Variável & $\begin{array}{l}\text { Grupo AVE } \\
\text { média } \pm \text { DP }\end{array}$ & $\begin{array}{l}\text { Grupo Controle } \\
\text { média } \pm \text { DP }\end{array}$ & $\begin{array}{c}p \text {-value } \\
\text { diferença média }(95 \% \text { IC) }\end{array}$ \\
\hline \multicolumn{4}{|c|}{ Caixa de blocos (quantidade por minutos) } \\
\hline Lado direito parético & $20,8 \pm 21,9$ & $69,7 \pm 12,1$ & $<0,001-48,9(-61,8--36,1)$ \\
\hline Lado direito não parético & $43,5 \pm 18,8$ & $69,7 \pm 12,1$ & $<0,001-26,2(-36,4--16,1)$ \\
\hline Lado esquerdo parético & $15,1 \pm 16,8$ & $58,5 \pm 25,8$ & $<0,001-43,4(-58,4--28,4)$ \\
\hline Lado esquerdo não parético & $45,5 \pm 15,1$ & $58,5 \pm 25,8$ & $0,195-12,9(-32,9-7,0)$ \\
\hline \multicolumn{4}{|l|}{ Dinamometria (KgF) } \\
\hline Lado direito parético & $22,4 \pm 27,0$ & $63,6 \pm 23,7$ & $<0,001-41,2(-62,0--20,4)$ \\
\hline Lado direito não parético & $48,3 \pm 20,6$ & $63,6 \pm 23,7$ & $0.045-15.3(-30,2--0,3)$ \\
\hline Lado esquerdo parético & $11,1 \pm 15,8$ & $59,7 \pm 23,2$ & $<0,001-48,6(-62,2--34,9)$ \\
\hline Lado esquerdo não parético & $47,8 \pm 22,3$ & $59,7 \pm 23,2$ & $0.223-11,8(-31,2-7,6)$ \\
\hline
\end{tabular}

\section{DISCUSSÃO}

Foi observado neste estudo que os indivíduos com sequela de AVE, apresentaram desempenho inferior ao grupo controle, com maior tempo de movimento, na realização da tarefa em todos os índices de dificuldade avaliados. No entanto, estes apresentaram comportamento semelhante aos indivíduos saudáveis ao longo da realização dos índices de dificuldade, com maior tempo de movimento naqueles que exigiam maior acurácia.

Este déficit do controle motor do grupo AVE é decorrente da sequela de AVE, causando redução da habilidade motora fina, essencial para tarefas cotidianas, as quais necessitam de velocidade e acurácia, planejamento e execução eficientes. ${ }^{35-39}$

Este fato do acometimento do controle motor fino, também foi enfatizado em um estudo ${ }^{40}$ que determinou o prejuízo da força de preensão palmar, da coordenação motora e da velocidade de movimento, como os principais padrões característicos de comprometimento no AVE. Em nosso estudo, também foi demonstrado essas mesmas características, ao verificarmos a destreza manual e coordenação motora com a caixa de blocos, e a força de preensão palmar com o uso do dinamômetro.

O mesmo declínio foi observado, com diferença significativa, tanto no membro superior parético direito e esquerdo, quanto no membro superior não parético direito, quando comparados com indivíduos saudáveis, ou seja, a lesão cerebral ocasionada pelo AVE, afeta o desempenho motor manual. Sendo um importante achado, a redução da força muscular e da função manual do membro contralateral, além do membro comprometido.

Essa fraqueza muscular presente nos indivíduos com a sequela, é provavelmente devido à perda das projeções diretas do trato corticoespinal do córtex motor para os circuitos motores da medula espinhal. ${ }^{41}$

Este córtex é o principal sistema motor para movimentos funcionais e geralmente encontra-se afetado pós AVE, ocasionando uma disfunção motora que provoca fraqueza muscular, paresia, coordenação de movimentos prejudicada, incluindo hiperreflexia e espasticidade. ${ }^{41}$

Sabe-se que quanto mais extensa a degeneração subsequente à lesão do trato corticoespinal que está intimamente relacionada aos déficits motores, mais fraca é a força de preensão e a destreza da mão e maior o nível de deficiência física. ${ }^{42-45}$ Entretanto, em relação a força de preensão palmar e função motora nos indivíduos pós-AVE neste estudo, não foi encontrada diferença significativa somente no lado esquerdo não parético, comparado a indivíduos sádios, mesmo com o achado do comprometimento do controle motor.

Quando o lado esquerdo do corpo é o não parético, significa que o lado comprometido pela hemiparesia (sequela do AVE) é o direito, por consequente acometimento do hemisfério esquerdo pela lesão sofrida no AVE.

Este achado pode ser justificado devido a área motora suplementar e o córtex pré-frontal no hemifério direito estarem preservados nestes indivíduos, já que nestas regiões em específico, há maior processamento da velocidade e precisão do movimento por um sistema de tomada de decisão que interage com circuitos neurais, e interferem na força muscular e na função. ${ }^{46,47}$

As sequelas neurológicas decorrentes do AVE e suas consequências, como a fraqueza muscular, interferiram na relação de velocidade e acurácia do movimento dos membros superiores neste estudo. Ao compararmos o ID2 com ID4a, em que há maior exigência de acurácia no ID4a, constatou-se aumento no tempo de movimento para execução da tarefa em ambos os grupos, porém o Grupo AVE foi mais lento em relação ao controle.

Em um outro estudo, ${ }^{48}$ que também avaliou pacientes com sequela de AVE pela Lei de Fitts, no entanto com uma tarefa diferente, esse padrão de movimento também foi encontrado, o qual sugere que o comprometimento da velocidade aumenta quando o indivíduo é obrigado a ajustar a velocidade a fim de manter precisão. 
Além disso, não foi encontrado efeito ou interação para os índices de dificuldade ID4a para ID4b (índices que apresentam o mesmo log-linear pela Lei de Fitts) para ambos os grupos, porém, este achado difere do encontrado em outro estudo, ${ }^{20}$ que ao comparar ID4a-ID4b em indivíduos com paralisia cerebral $(\mathrm{PC})$ com indivíduos saudáveis, encontrou diferença significativa entre ambos, sugerindo que o grupo com PC não obedeceu à relação entre tamanho e distância dos alvos (lei de Fitts), com maior tempo de movimento no ID4b, diferentemente do que foi evidenciado em nosso estudo.

Sendo assim, indivíduos com sequelas neurológicas podem não ter o mesmo padrão de comprometimento do controle motor, porém em ambos os casos, persistiu-se comportamento do maior tempo de movimento destes, em relação a indivíduos saudáveis.

Comparando os resultados de ID4b (média $=3,318 \mathrm{~s}$ ) com ID6 (média $=4,132 \mathrm{~s}$ ) entre os grupos estudados, o mesmo comportamento gradual foi observado. Sendo que o Grupo AVE realizou a tarefa do ID6 em maior tempo, enquanto o controle necessitou de menor tempo para executá-la, ou seja, indivíduos com AVE, quando há exigência de mais acurácia em uma tarefa, apresentam maior dificuldade e necessitam de mais tempo.

Assim como demonstrou outro estudo, ${ }^{49}$ que utilizou a realidade virtual (RV) imersiva com dispositivo 3D para avaliar extremidade superior em indivíduos com AVE, os quais tinham que executar uma tarefa de apontar alvos que surgiam e desapareciam. Foi visto que o grupo com AVE teve significativamente maior tempo de movimento quando comparado ao grupo controle, assim como demonstrado em nosso estudo.

Confirmando que após o AVE, os indivíduos necessitam de mais tempo para executar uma tarefa que exige controle motor (velocidade e acurácia) em um ambiente de realidade virtual imersiva e não imersiva. Porém, o estudo não cita se os indivíduos pós-AVE avaliados apresentaram maior acometimento de velocidade ou de acurácia.

Já em outro estudo, ${ }^{39}$ que avaliou os mecanismos comportamentais e neurofisiológicos subjacentes à habilidade motora e déficits na aprendizagem com o braço parético, resultou em diferenças de acurácia entre o grupo controle e o grupo AVE.

Apesar de realizarem o que foi solicitado com seu desempenho máximo, os pacientes com AVE demonstraram déficits significativos no desempenho de habilidades, particularmente quando colocados frente a um maior desafio (por exemplo, em alvos menores).

Quando realizaram tarefas em que os alvos eram maiores não houve diferença estatística na acurácia entre o controle e os indivíduos com $\mathrm{AVE},{ }^{39}$ achado que difere ao deste estudo, no qual o Grupo AVE apresentou maior tempo de movimento do que o controle em todos os índices de dificuldade, porém com menor tempo de movimento naqueles com as barras eram mais largas, demonstrando maior déficit na acurácia.

Além disso, os autores citados $^{39}$ descreveram em seu estudo, que ao praticar tarefas que exijam habilidades complexas, o sistema corticoespinhal ipsilesional em pacientes com AVE de grau leve e moderado, pode ser capaz de reorganizar-se para suportar a performance no braço parético.
A lei de Fitts determina a relação inversamente proporcional entre a velocidade e a acurácia do movimento, na qual quanto maior a velocidade, menor acurácia e vice- versa, desta forma fisiologicamente ocorre em todos os indivíduos.

Porém, na sequela de AVE, além desta situação fisiológica, temos fatores de coordenação e interação visuomotora comprometidos, os quais um outro estudo, ${ }^{46}$ determinou que com a modulação extracorpórea realizada pelos estímulos e a atenção visual do indivíduo, sobre a região cortical e subcortical, demonstra que estes pré-estímulos para a realização da atividade que exige acurácia pode ser afetada, ocasionando a sua piora nas ações pós- estímulo. ${ }^{50,51}$ Sugerese também que há um atraso no processamento da informação e execução da atividade, devido a uma diminuição da velocidade de informação neuronal e da coordenação visuomotora. ${ }^{52,53}$

Além do indivíduo pós AVE apresentar maior tendência de erros, diminuindo o controle e gerando movimentos mais segmentados, como consequência estes indivíduos são mais lentos para alcançar a acurácia exigida no movimento. ${ }^{54}$ Fatores estes que prejudicaram a execução da tarefa realizada neste estudo.

Como o comprometimento motor é um dos principais fatores que influenciam o bem-estar um ano após o AVE, ${ }^{55}$ melhorar a função do membro superior é, portanto, um elemento central da reabilitação, a fim de maximizar os resultados do paciente, melhorar o bem-estar e reduzir a incapacidade, ${ }^{56,57}$ o que demonstra a importância de estudos que analisem os movimentos de membro superior e as suas características, como o controle motor, para que haja melhor organização do processo de reabilitação e melhora do quadro destes pacientes.

\section{CONCLUSÃO}

Portanto, pode-se concluir neste estudo que os indivíduos com sequela de AVE apresentaram déficit do controle motor de membro superior, comparado a indivíduos saudáveis, porém estes apresentaram o mesmo comportamento, com déficit maior na acurácia do movimento. Esses achados direcionam para adequação de tarefas de motricidade fina na reabilitação destes indivíduos, para que estas envolvam e requeiram a velocidade e a acurácia do movimento, mas principalmente com foco nesta última. Já que são essenciais para execução de habilidades motoras no cotidiano dos indivíduos, e decorrente a sequela, sabe-se que há o acometimento no desempenho destas, não sendo geralmente o foco dos processos de reabilitação nesta população.

\section{AGRADECIMENTOS}

Agradecemos a Universidade do Oeste Paulista (UNOESTE) por todo apoio científico e pelo financiamento da pesquisa.

\section{REFERÊNCIAS}

1. Astor BC, Shafi T, Hoogeveen RC, Matsushita K, Ballantyne CM, Inker LA, et al. Novel markers of kidney function as predictors of ESRD, cardiovascular disease, and mortality in the general population. Am J Kidney Dis. 2012;59(5):653-62. Doi: https://doi.org/10.1053/j.ajkd.2011.11.042 
2. Mahmoodi BK, Yatsuya H, Matsushita K, Sang Y, Gottesman RF, Astor BC, et al. Association of kidney disease measures with ischemic versus hemorrhagic strokes: pooled analyses of 4 prospective community-based cohorts. Stroke. 2014;45(7):1925-31.

Doi:

https://doi.org/10.1161/STROKEAHA.114.004900

3. Brasil. Ministério da Saúde. Diretrizes de atenção à reabilitação da pessoa com acidente vascular cerebral. Brasília: Ministério da Saúde; 2013.

4. Botelho TS, Machado CD, Araújo NFLC, Assis SC. Epidemiologia do acidente vascular cerebral no Brasil. Temas em Saúde. 2016;16(2):361-377.

5. Langhorne P, Bernhardt J, Kwakkel G. Stroke rehabilitation. Lancet. 2011;377(9778):1693-702. Doi: https://doi.org/10.1016/S0140-6736(11)60325-5

6. Karahan AY, Kucuksen S, Yilmaz H, Salli A, Gungor T, Sahin M. Effects of rehabilitation services on anxiety, depression, caregiving burden and perceived social support of stroke caregivers. Acta Medica (Hradec Kralove). 2014;57(2):68-72. Doi: https://doi.org/10.14712/18059694.2014.42

7. Nakashima A, Moriuchi T, Mitsunaga W, Yonezawa T, Kataoka $H$, Nakashima $R$, et al. Prediction of prognosis of upperextremity function following stroke- related paralysis using brain imaging. J Phys Ther Sci. 2017;29(8):1438-43. Doi: https://doi.org/10.1589/jpts.29.1438

8. Go AS, Mozaffarian D, Roger VL, Benjamin EJ, Berry JD, Blaha MJ, et al. Heart disease and stroke statistics--2014 update: a report from the American Heart Association. Circulation. 2014;129(3):e28-e292.

https://doi.org/10.1161/01.cir.0000441139.02102.80

Doi:

9. Swanson LR, Whittinghill DM. Intrinsic or extrinsic? using videogames to motivate stroke survivors: a systematic review. Games Health J. 2015;4(3):253-8. Doi: https://doi.org/10.1089/g4h.2014.0074

10. Schieber $\mathrm{MH}$, Poliakov AV. Partial inactivation of the primary motor cortex hand area: effects on individuated finger movements. J Neurosci. 1998;18(21):9038-54. Doi: https://doi.org/10.1523/JNEUROSCI.18-21-09038.1998

11. Schieber $\mathrm{MH}$. Constraints on somatotopic organization in the primary motor cortex. J Neurophysiol. 2001;86(5):2125-43. Doi: https://doi.org/10.1152/jn.2001.86.5.2125

12. Nakayama H, Jørgensen HS, Raaschou HO, Olsen TS. Compensation in recovery of upper extremity function after stroke: the Copenhagen Stroke Study. Arch Phys Med Rehabil. 1994;75(8):852-7. Doi: https://doi.org/10.1016/00039993(94)90108-2

13. Kim Y, Kim WS, Shim JK, Suh DW, Kim T, Yoon B. Difference of motor overflow depending on the impaired or unimpaired hand in stroke patients. Hum Mov Sci. 2015;39:154-62. Doi: https://doi.org/10.1016/j.humov.2014.11.007

14. Térémetz M, Colle F, Hamdoun S, Maier MA, Lindberg PG. A novel method for the quantification of key components of manual dexterity after stroke. J Neuroeng Rehabil. 2015;12:64. Doi: https://doi.org/ 10.1186/s12984-015-0054-0

15. Kim Y, Kim WS, Yoon B. The effect of stroke on motor selectivity for force control in single- and multi-finger force production tasks. NeuroRehabilitation. 2014;34(3):429-35. Doi: https://doi.org/10.3233/NRE-141050

16. Paquin K, Ali S, Carr K, Crawley J, McGowan C, Horton S. Effectiveness of commercial video gaming on fine motor control in chronic stroke within community-level rehabilitation. Disabil Rehabil. 2015;37(23):2184-91. Doi: https://doi.org/10.3109/09638288.2014.1002574
17. Roh J, Rymer WZ, Beer RF. Evidence for altered upper extremity muscle synergies in chronic stroke survivors with mild and moderate impairment. Front Hum Neurosci. 2015;9:6. Doi: https://doi.org/10.3389/fnhum.2015.00006

18. Kim Y, Kim WS, Koh K, Yoon B, Damiano DL, Shim JK. Deficits in motor abilities for multi-finger force control in hemiparetic stroke survivors. Exp Brain Res. 2016;234(8):2391-402. Doi: https://doi.org/10.1007/s00221-016-4644-2

19. Ifft PJ, Lebedev MA, Nicolelis MA. Cortical correlates of fitts' law. Front Integr Neurosci. 2011;5:85. Doi: https://doi.org/10.3389/fnint.2011.00085

20. Fernani DCGL, Prado MTA, Silva TD, Massetti T, Abreu LC, Magalhães $\mathrm{FH}$, et al. Evaluation of speed-accuracy trade-off in a computer task in individuals with cerebral palsy: a crosssectional study. BMC Neurol. 2017;17(1):143. Doi: https://doi.org/10.1186/s12883-017-0920-4

21. Davies TC, AIManji A, Stott NS. A cross-sectional study examining computer task completion by adolescents with cerebral palsy across the Manual Ability Classification System levels. Dev Med Child Neurol. 2014;56(12):1180-1186. Doi: https://doi.org/10.1111/dmcn.12521

22. Prado MTA, Fernani DCGL, Silva TDD, Smorenburg ARP, Abreu LC, Monteiro CBM. Motor learning paradigm and contextual interference in manual computer tasks in individuals with cerebral palsy. Res Dev Disabil. 2017;64:56-63. Doi: https://doi.org/10.1016/j.ridd.2017.03.006

23. Folstein MF, Folstein SE, McHugh PR. "Mini-mental state". A practical method for grading the cognitive state of patients for the clinician. J Psychiatr Res. 1975 Nov;12(3):189-98. Doi: https://doi.org/10.1016/0022-3956(75)90026-6

24. Rieck M, Moreland J. The Orpington Prognostic Scale for patients with stroke: reliability and pilot predictive data for discharge destination and therapeutic services. Disabil Rehabil. 2005;27(23):1425-33. https://doi.org/10.1080/09638280500330435

25. Fugl-Meyer AR, Jääskö L, Leyman I, Olsson S, Steglind S. The post-stroke hemiplegic patient. 1. a method for evaluation of physical performance. Scand J Rehabil Med. 1975;7(1):13-31.

26. Maki T, Quagliato E, Cacho E, Paz L, Nascimento NH, Inoue M, et al. Estudo de confiabilidade da aplicação da escala de FuglMeyer no Brasil. Rev Bras Fisioter. 2006;10(2):177-83. Doi: https://doi.org/10.1590/S1413-35552006000200007

27. Guimarães R, Blascovi-Assis SM. Uso do teste caixa e blocos na avaliação de destreza manual em crianças e jovens com síndrome de Down. Rev Ter Ocup Univ São Paulo. 2012: (23):98-106. Doi: https://doi.org/10.11606/issn.22386149.v23i1p98-106

28. Mathiowetz V, Weber K, Volland G, Kashman N. Reliability and validity of grip and pinch strength evaluations. J Hand Surg Am. 1984;9(2):222-6. Doi: https://doi.org/10.1016/s03635023(84)80146-x

29. Mathiowetz V. Grip and pinch strength measurements. In: Amudsen LR (ed). Muscle strength testing: instrumented and non-instrumented systems. New York: Churchill Livingstone; 1990. p.163-75.

30. Stephens JL, Pratt N, Parks B. The reliability and validity of the Tekdyne hand dynamometer: Part I. J Hand Ther. 1996;9(1):107. Doi: https://doi.org/10.1016/s0894-1130(96)80006-8

31. Okazaki VHA. Discrete Aiming Task [computer program]. Version 2.02007 [cited 2017 Out 18]. Available from: http://okazaki.webs.com/softwaresdownloads.htm\#2970515 $\underline{18}$ 
32. Mathiowetz V, Volland G, Kashman N, Weber K. Adult norms for the Box and Block Test of manual dexterity. Am J Occup Ther. 1985;39(6):386-91.

Doi: https://doi.org/10.5014/ajot.39.6.386

33. Son BY, Bang YS, Hwang MJ, Oh EJ. Effect of task-oriented activities on hand functions, cognitive functions and selfexpression of elderly patients with dementia. J Phys Ther Sci. 2017;29(8):1357-1362.

https://doi.org/10.1589/jpts.29.1357

Doi:

34. Fitts PM. The information capacity of the human motor system in controlling the amplitude of movement. 1954. J Exp Psychol Gen. 1992;121(3):262-9.

Doi:

http://dx.doi.org/10.1037//0096-3445.121.3.262

35. Begliomini C, De Sanctis T, Marangon M, Tarantino V, Sartori L, Miotto $D$, et al. An investigation of the neural circuits underlying reaching and reach-to-grasp movements: from planning to execution. Front Hum Neurosci. 2014;8:676. Doi: https://doi.org/10.3389/fnhum.2014.00676

36.Fang Y, Daly JJ, Hansley J, Yao WX, Yang Q, Sun J, et al. Hemispheric activation during planning and execution phases in reaching post stroke: a consort study. Medicine (Baltimore). 2015;94(3):e307.

Doi: https://doi.org/10.1097/MD.0000000000000307

37. Orban de Xivry JJ, Legrain V, Lefèvre P. Overlap of movement planning and movement execution reduces reaction time. J Neurophysiol. 2017;117(1):117-22.

Doi: https://doi.org/10.1152/in.00728.2016

38. Stewart JC, Gordon J, Winstein CJ. Control of reach extent with the paretic and nonparetic arms after unilateral sensorimotor stroke: kinematic differences based on side of brain damage. Exp Brain Res. 2014;232(7):2407-19. Doi: https://doi.org/10.1007/s00221-014-3938-5

39. Kantak S, McGrath R, Zahedi N, Luchmee D. Behavioral and neurophysiological mechanisms underlying motor skill learning in patients with post-stroke hemiparesis. Clin Neurophysiol. 2018;129(1):1-12.

Doi: https://doi.org/10.1016/j.clinph.2017.10.010

40. Allgöwer K, Hermsdörfer J. Fine motor skills predict performance in the Jebsen Taylor Hand Function Test after stroke. Clin Neurophysiol. 2017;128(10):1858-71. Doi: https://doi.org/10.1016/j.clinph.2017.07.408

41. Williams PTJA, Jiang YQ, Martin JH. Motor system plasticity after unilateral injury in the developing brain. Dev Med Child Neurol. 2017;59(12):1224-29. Doi: https://doi.org/10.1111/dmcn.13581

42. Lindberg PG, Skejø PH, Rounis E, Nagy Z, Schmitz C, Wernegren $\mathrm{H}$, et al. Wallerian degeneration of the corticofugal tracts in chronic stroke: a pilot study relating diffusion tensor imaging, transcranial magnetic stimulation, and hand function. Neurorehabil Neural Repair. 2007;21(6):551-60. Doi: https://doi.org/10.1177/1545968307301886

43. Borich MR, Mang C, Boyd LA. Both projection and commissural pathways are disrupted in individuals with chronic stroke: investigating microstructural white matter correlates of motor recovery. BMC Neurosci. 2012;13:107. Doi: https://doi.org/10.1186/1471-2202-13-10

44. Tuszynski MH, Steward O. Concepts and methods for the study of axonal regeneration in the CNS. Neuron. 2012;74(5):777-91. Doi: https://doi.org/10.1016/j.neuron.2012.05.006
45. Zhang C, Zou Y, Li K, Li C, Jiang Y, Sun J, et al. Different effects of running wheel exercise and skilled reaching training on corticofugal tract plasticity in hypertensive rats with cortical infarctions. Behav Brain Res. 2018;336:166-72. Doi: https://doi.org/10.1016/j.bbr.2017.09.002

46. Perri RL, Berchicci M, Spinelli D, Di Russo F. Individual differences in response speed and accuracy are associated to specific brain activities of two interacting systems. Front Behav Neurosci. 2014;8:251.

Doi: https://doi.org/10.3389/fnbeh.2014.00251

47. O'Regan L, Serrien DJ. Individual differences and hemispheric asymmetries for language and spatial attention. Front Hum Neurosci. 2018;12:380.

Doi: https://doi.org/10.3389/fnhum.2018.00380

48. Haaland KY, Prestopnik JL, Knight RT, Lee RR. Hemispheric asymmetries for kinematic and positional aspects of reaching. Brain. 2004;127(Pt 5):1145-58. Doi: https://doi.org/10.1093/brain/awh133

49. Hussain N, Alt Murphy M, Sunnerhagen KS. Upper Limb Kinematics in stroke and healthy controls using target-totarget task in virtual reality. Front Neurol. 2018;9:300. Doi: https://doi.org/10.3389/fneur.2018.00300

50. Mesulam MM. Large-scale neurocognitive networks and distributed processing for attention, language, and memory. Ann Neurol. 1990;28(5):597-613. Doi: https://doi.org/10.1002/ana.410280502

51. Nobre AC, Sebestyen GN, Gitelman DR, Mesulam MM, Frackowiak RS, Frith CD. Functional localization of the system for visuospatial attention using positron emission tomography. Brain. 1997;120 (Pt3):515-33. Doi: https://doi.org/10.1093/brain/120.3.515

52. Rae B, Heathcote A, Donkin C, Averell L, Brown S. The hare and the tortoise: emphasizing speed can change the evidence used to make decisions. J Exp Psychol Learn Mem Cogn. 2014;40(5):1226-43. Doi: https://doi.org/10.1037/a0036801

53. Zhang J, Rowe JB. Dissociable mechanisms of speed-accuracy tradeoff during visual perceptual learning are revealed by a hierarchical drift-diffusion model. Front Neurosci. 2014;8:69. Doi: https://doi.org/10.3389/fnins.2014.00069

54. Mottet D, van Dokkum LE, Froger J, Gouaïch A, Laffont I. Trajectory formation principles are the same after mild or moderate stroke. PLoS One. 2017;12(3):e0173674. Doi: https://doi.org/10.1371/journal.pone.0173674

55. Wyller TB, Holmen J, Laake P, Laake K. Correlates of subjective well-being in stroke patients. Stroke. 1998;29(2):363-7. Doi: https://doi.org/10.1161/01.str.29.2.363

56. Langhorne P, Legg L. Evidence behind stroke rehabilitation. J Neurol Neurosurg Psychiatry. 2003;74 Suppl 4(Suppl 4):iv18iv21. Doi: http://dx.doi.org/10.1136/jnnp.74.suppl_4.iv18

57. Coupar F, Pollock A, Legg LA, Sackley C, van Vliet P. Homebased therapy programmes for upper limb functional recovery following stroke. Cochrane Database Syst Rev. 2012;2012(5):CD006755. https://doi.org/10.1002/14651858.CD006755 